\title{
Comparative Study Using Functional and Stabilometric Evaluation of Balance in Elderly Submitted to Conventional Physiotherapy and Wii- rehabilitation
}

\author{
João Paulo Alves do Couto ${ }^{1 *}$, Maiama Leite do Nascimento ${ }^{2}$ and Osmar Pinto Neto ${ }^{3}$ \\ ${ }^{1}$ Department of Biomedical Engineering, University Camilo Castelo Branco: Parque Tecnologico de Sao Jose dos Campos, Brazil \\ 2Integrated College of Southern Bahia, Brazil \\ ${ }^{3}$ Department of Physiology and Kinesiology, São José dos Campos - SP Brazil
}

\begin{abstract}
Objective: To analyze and compare the benefits and advantages of using the Nintendo® WII console software and conventional physiotherapy for improving the balance in the elderly.

Materials and methods: Sixteen elders, divided in 2 groups according to the received treatment (conventional physiotherapy or Wii-based rehabilitation), had their balance evaluated by 4 methods, by using the Berg balance scale, the functional reach, the "time up and go" test, and stabilometry.

Results: Berg balance scale scores improved for the Wii-based rehabilitation group. Larger body displacement towards the frontal region was observed in the functional reach test for the participants treated with Wii-based rehabilitation. Decreased time for completing the "time up and go" test was observed for Wii-based rehabilitation. Stabilometric data revealed no significant differences between the two groups or between the different evaluations.
\end{abstract}

Conclusions: We conclude that the Wii-based rehabilitation treatment was more effective in improving balance functional parameters than a more traditional physiotherapy protocol for older adults.

Keywords: Balance; Physiotherapy; Wii-based rehabilitation; Elderly

\section{Introduction}

Balance can be defined as the ability of an individual to successfully maintain his/her body posture or, more specifically, his/her center of gravity within the physical limits of space. Balance maintenance can be static (remaining at rest) or dynamic (during motion), and depends on a complex interaction between the sensory and musculoskeletal systems [1].

Biological aging is associated with profound changes in cellular, tissue-level, and organic activities, resulting in a reduction in the efficiency of several physiological processes of the human body. Among the physiological processes that exhibit age-related deterioration is the ability to maintain body balance, which leads to postural abnormalities, postural instability and falls. Different tests can be used for diagnosis in patients with balance disorders [2-4].

Melzer et al. [5] pointed out that aging contributes to the deterioration of balance control mechanisms, causing balance disparities, which leads to the occurrence of falls and limited functionality.

Many mechanisms have been proposed to explain age-related changes in balance. Assuming that aging affects all physiological processes, postural balance reduction can be explained by the loss of cellular receptors in the vestibular system, impaired sensory perception, decreased muscular strength, increased reaction time [5] and changes in proprioception [6].

Reduced physical activity can also cause a reduction in balance in the elderly. Balance disorders represent a growing public health concern, owing to their connection with falls and the associated consequences. These consequences include fractures and immobilization or prolonged hospitalization, leading to losses in autonomy and quality of life of the elderly $[7,8]$.

These considerations stress the need to develop a program of special corporal perception movements for enhancing the reorganization of body balance during aging, before the loss of balance and consequent falls lead to social withdrawal, with serious physical and mental consequences for the elderly [9].

According to Vaghetti et al. [10], state-of-the-art technology has been widely used in several health care areas, in particular for the recovery of cognitive and motor autonomy. Physiotherapy, in general, makes use of diverse methods for recovering the motor abilities of the injured individual and, as a consequence, aids in improving his/her self-esteem. Virtual learning environments are gaining wide use, and videogames are increasingly adopted for working on sensory and motor skills.

Butler et al. [11] note that virtual environments encourage highintensity repetition and have been used in numerous professional training programs. Current rehabilitation programs are also employing these tools by using virtual reality (e.g., Nintendo ${ }^{\circ}$ Wii). The Nintendo Wii is the seventh generation video game console developed and sold by Nintendo. Its unique feature is the ability to project the physical movement of its user onto the TV screen, which increases interactivity and user's stimulus to play the game, thus providing the user with a

*Corresponding author: João Paulo Alves do Couto, Department of Biomedical Engineering, University Camilo Castelo Branco: Parque Tecnologico de Sao Jose dos Campos, Road Doctor Altino Bondesan, 500, Melo Eugenius-São José dos Campos-SP Brazil, Tel: 557381531743; E-mail: jcouto@unece.br

Received: March 01, 2016; Accepted: May 04, 2016; Published: May 10, 2016

Citation: do Couto JPA, do Nascimento ML, Neto OP (2016) Comparative Study Using Functional and Stabilometric Evaluation of Balance in Elderly Submitted to Conventional Physiotherapy and Wii-Rehabilitation. Physiother Rehabil 1: 109. doi:10.4172/2573-0312.1000109

Copyright: ( 2016 do Couto JPA, et al. This is an open-access article distributed under the terms of the Creative Commons Attribution License, which permits unrestricted use, distribution, and reproduction in any medium, provided the original author and source are credited. 
quick feedback of the movement being performed. The user has a physical sensation, related to the interaction with objects. Although this sensation may not be identical to a sensation perceived when interacting with objects in the real world, it is enhanced by auditory feedback. The Wii console uses the differences between applied force and acceleration components on its controls to adjust the amount of feedback provided to the user.

Thus, the Wii system offers clinicians the potential to develop routines for practicing both fine and gross motor skills, for coordination and motor control, and for improving the patient balance by using the Wii balance board $[12,13]$.

The goal of this study was to analyze and compare the benefits and advantages of using the Nintendo ${ }^{\circ}$ WII game console software and conventional physiotherapy for improving the balance in the elderly.

\section{Materials and Methods}

\section{Ethical aspects}

This study conformed to the ethical principles and followed the national and international guidelines for research involving human beings (CAAE, Protocol no 01092112.5.0000.5062).

\section{Experimental model}

Sixteen elderly residents of the city of Eunápolis BA, 9 females and 7 males, all over 60 years old, provided their informed consent to be included in the study.

The 16 elderly patients were randomly divided into two groups, with $\mathrm{n}=8$ for each group:

The conventional physiotherapy group included the elderly who were treated by using physiotherapy and were evaluated periodically.

The Wii-based rehabilitation group included the elderly who participated in guided activities by using the Nintendo ${ }^{\circ}$ Wii game console and were evaluated periodically.

\section{Therapeutic procedures}

The elderly patients of the physiotherapy and Wii-based rehabilitation groups were submitted to three weekly sessions with the respective protocols, for a total of 12 sessions. Each treatment session lasted 40-50 min. The procedures took place in the school clinic of UnisulBahia Faculdades Integradas. The elderly in the control group were only evaluated, without performing any treatment. All elderly patients were evaluated during the $1^{\text {st }}, 6^{\text {th }}$, and $12^{\text {th }}$ sessions.

Therapeutic procedure using conventional physiotherapy: The elderly treated by using conventional physiotherapy had to perform the following therapeutic activities: stretching exercises, strengthening, weight transfer, body stability and marching in three series of ten repetitions [14-22].

The stretching exercises were performed passively for the quadriceps, hamstrings, triceps surae, adductors and abductors [14$16,22]$. Strengthening amounted to performing squatting exercises $[16,21,22]$. Weight transfer exercises were performed as follows: the patient and the physiotherapist stood back to back, a ball was passed by hand, with trunk rotation associated with the elevation of the lower ipsilateral member, ten passages for each side [14]. Body stability tasks were performed with normal base, standing with closed eyes, with both feet parallel to each other, followed by manual disturbance, standing on an instable surface (proprioceptive disk) and throwing a ball up in the air and catching it without letting it fall to the ground [19,20]. Marching tasks were performed with the normal base. The patients were asked to walk over a straight line on the ground, in parallel bars [19,21,22].

Therapeutic procedure using wii-based rehabilitation: The exercises involving the Wii Fit Plus software, combined with the Wii Balance Board, were used as a therapy. The games used were: Lotus Focus, also known as „Zazen”: The participant was in the orthostatic position, on the Wii Balance Board, with his/her feet a shoulder width apart. When the game started, the participant had to remain still for as long as possible, while breathing normally.

Deep breathing: The participant was in the orthostatic position, with his/her feet a shoulder width apart, on the Wii Balance Board, with his/her hands positioned over the abdomen, near the belly button. The participant inhaled and exhaled slowly while trying to maintain a red dot at the center of a yellow balance line on the screen.

Penguin slide: The participant was in the orthostatic position, with his/her feet a shoulder width apart, on the Wii Balance Board, performing weight transfer changes to the left and to the right to tilt the iceberg in order to capture the fish. By tilting the iceberg, the avatar started to slide down in the direction of the inclination. If the participant was not careful, the avatar slid off the iceberg and the participant had to wait a few seconds before the avatar could be re-allowed to pick up fish.

Balance bubble: The participant was in the orthostatic position, with his/her feet a shoulder width apart, on the Wii Balance Board. In this game, the participant had to perform a weight transfer task to the anterior region for the avatar to move forward or, in the case, he/ she wanted the avatar to move backwards, to the posterior region. The participant also performed weight transfers to the left and to the right, for moving the avatar to conform to the weight transfer.

Table tilt: In this game, the participant had to place a set of balls in holes on a platform. To do so, the participant was in the orthostatic position, with feet a shoulder width apart on the Wii Balance Board, and performed a weight transfer task to the anteroposterior, right and left regions to tilt the floating platform and guide the balls into the holes. Each exercise was performed at least five times.

\section{Balance analysis}

Baropodometry, Berg balance scale, functional reach test and "time up and go" test were applied for the balance analysis. For all participants, the tests were performed during the $1^{\text {st }}, 6^{\text {th }}$, and $12^{\text {th }}$ sessions.

Baropodometry: We used an electronic baropodometer of the Arkipelago brand and a platform guided by the Footwork program. Data were acquired for performing baropodometric bipodalic analysis, through posturographic analysis barefoot and with open eyes. The statokinesiogram and the stabilogram were used as comparison parameters. The statokinesiogram is the map of the center of pressure (CP) in the anteroposterior direction versus the $\mathrm{CP}$ in the mediolateral direction. The stabilogram is the temporal series of the $\mathrm{CP}$ in each direction: anteroposterior and mediolateral [23].

Several time-and frequency-domain variables were calculated from the $\mathrm{CP}$ displacement data. In the time domain the calculated variables were: the dispersion of the Center of Pressure displacement from the mean position in the anteroposterior direction (SDap); the dispersion of the Center of Pressure displacement from the mean position in the mediolateral direction (SDml); the root mean square displacement in the anteroposterior direction (RMSap); the root mean square displacement in the mediolateral direction (RMSml); the total 
average speed (TAS); the maximal and minimal CP displacements in the anteroposterior direction (AdCPap), and the maximal and minimal $\mathrm{CP}$ displacements in the mediolateral direction (AdCPml).

Berg balance scale: The Berg balance scale test consisted of 14 tasks, on an ordinal scale of 4 points, ranging from 0 (incapable of performing the task) to 4 (performs the task autonomously). The scores for the 14 tasks were summed for obtaining a total score, which varied between 0 and 56 points. A high score implied better performance and values equal or lower than 45 predicted the risk of fall [3]. Thorbann and Newton [24] add to this, reporting high specificity (96\%) and low sensitivity (53\%) for this test, for the predisposition value of 45 points.

Functional reach test: This test determined to what extent the elderly were capable of moving within the anterior stability limit, with their feet being parallel. Duncan et al. [25] have shown that individuals who were able to reach within 25.4 to $15.2 \mathrm{~cm}$ were twice more likely to suffer two or more falls in the next 6 months, when compared with those who could reach $25.4 \mathrm{~cm}$ or more; those who reached for $15.2 \mathrm{~cm}$ or less were 4 times more likely to suffer two or more falls in the next 6 months.

"Time up and go" test (TUG): This test quantified the time (in seconds) that the individual required to rise from a standardized chair, with arm rest and $46 \mathrm{~cm}$ above the floor, walk $3 \mathrm{~m}$, turn, walk back to the chair, and sit down. Bohannon [26] performed a meta-analysis, revealing that the average time to complete this "time up and go" test was $9.4 \mathrm{~s}$ for elderly. It was also shown that this time increases with age. Thus, using the upper limit of the confidence interval it is possible to say that performance time longer than $9 \mathrm{~s}$ for elderly between 60 and 69 years old, longer than $10.2 \mathrm{~s}$ for elderly between 70 and 79 years old, and $12.7 \mathrm{~s}$ for elderly between 80 and 99 years old, can be considered worse than the average for the respective age groups. Elders who completed the "time up and go" test in more than $13.5 \mathrm{~s}$ have an increased risk of fall.

\section{Statistical analysis}

The Bioestat 5.0 software was used for statistical analysis. After the descriptive analysis of the data, the Shapiro-Wilk test was used to assess the normality of the data distribution. As variables were shown to be non-parametric, the Wilcoxon test was used to compare variables within each group and the Mann-Whitney test was used for comparisons between the groups. A significance level of $p<0.05$ was adopted for all analyses.

\section{Results}

The results suggest that, at the end of the 12 sessions, the elderly who received the treatment with Wii-based rehabilitation obtained better Berg scores than the elderly in the conventional physiotherapy group. The subjects of the Wii-based rehabilitation group obtained an average score 46.12 points $( \pm 1.20)$ and the conventional physiotherapy group obtained 45.12 points $( \pm 1.60)$ at the first evaluation. At the end of the third evaluation, the Wii-based rehabilitation group obtained an average score 53.75 points $( \pm 1.84)$ and the conventional physiotherapy group obtained 50.25 points $( \pm 1.35)$. Comparing the results of the third evaluation between the Wii-based rehabilitation and the conventional physiotherapy group, we found that the Wii-based rehabilitation group exhibited significantly $(\mathrm{p}=0.04)$ higher Berg balance scale scores. In addition, only the Wii-based rehabilitation group exhibited a significant $(\mathrm{p}=0.01)$ increase in scores from the first to the third evaluation (Figure 1).

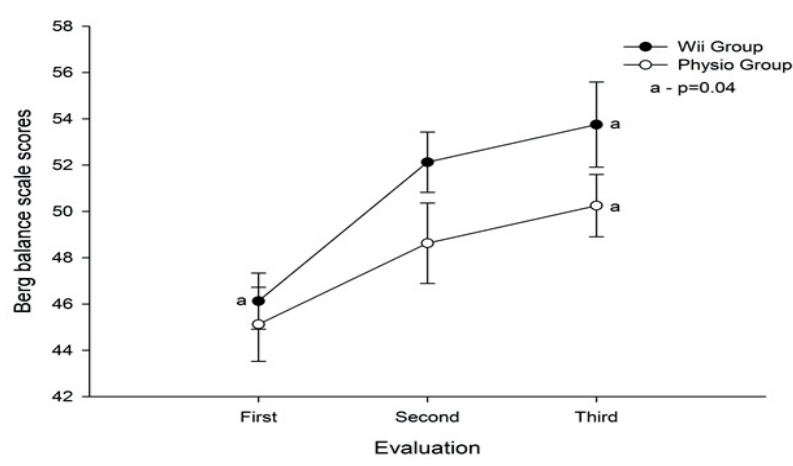

Figure 1: The Berg balance scale scores, for the Wii-based rehabilitation and conventional physiotherapy groups, for the $1^{\text {st }}, 2^{\text {nd }}$ and $3^{\text {rd }}$ evaluations.

Additionally, the results of the functional reach test showed that the subjects of the Wii-based rehabilitation group obtained an average reach of $23 \mathrm{~cm}( \pm 2.26)$ and the conventional physiotherapy group obtained an average reach of $25.12 \mathrm{~cm}( \pm 1.99)$. At the end of the third evaluation, the subjects of the Wii-based rehabilitation group obtained an average reach of $29.75 \mathrm{~cm}( \pm 1.33)$ and the conventional physiotherapy group obtained an average reach of $23.62 \mathrm{~cm}( \pm 2.25)$. The Wii-based treatment group was the only one in which the elderly were positively and significantly affected by the treatment. In this group, the elderly exhibited a significant $(\mathrm{p}=0.01)$ increase in reach in the third evaluation, compared with the first evaluation. Moreover, in the third evaluation, the Wii-based rehabilitation group exhibited significantly $(\mathrm{p}=0.03)$ higher scores than the conventional physiotherapy group (Figure 2).

For the TUG test, we found that the Wii-based rehabilitation group performed an average of $10.37 \mathrm{~s}( \pm 0.86)$ while the conventional physiotherapy group performed in $12.75( \pm 2.60)$. By the end of the third evaluation, the subjects of the Wii-based rehabilitation group performed an average of 8 seconds $( \pm 0.70)$ and the conventional physiotherapy group in $10 \mathrm{~s}( \pm 1.48)$. The only statistically significant difference $(p=0.03)$ was obtained for the comparison of the first and third evaluations of the Wii-based rehabilitation group, for which the elderly performed $2 \mathrm{~s}$ faster in the latter (Figure 3 ).

The stabilometric data revealed no statistically significant differences between the two groups or between the different evaluations (Table 1).

\section{Discussion}

Our study shows that both conventional physiotherapy and Wiibased rehabilitation help to improve and/or maintain balance in the elderly. The results show the following: (1) a significant improvement in the Berg balance scale scores in Wii-based rehabilitation groups; (2) larger body displacement in the frontal direction in the functional reach test for the participants in the Wii-based rehabilitation group; (3) smaller times for the "time up and go" test for both groups; (4) no differences were observed for the evaluated stabilometric variables.

Evaluation by using the Berg balance scale revealed a significant improvement of balance, with statistical significance of $p<0.05$, between the first and third sessions, for the Wii-based rehabilitation group. In addition, a statistically significant difference was observed when comparing the results for the Wii-based rehabilitation and conventional physiotherapy groups at the end of the third evaluation. 


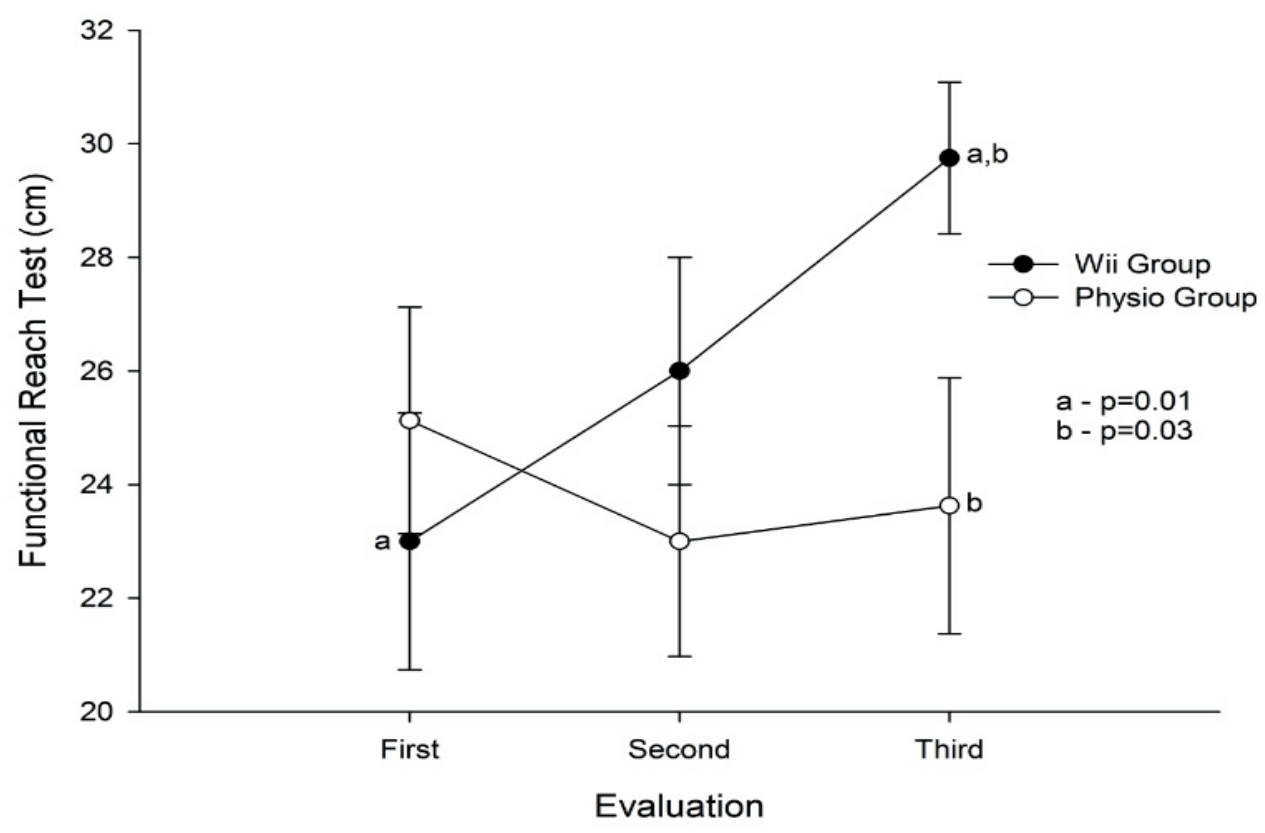

Figure 2: Distance in centimeters in the functional reach test, for the Wii-based rehabilitation and conventional physiotherapy groups, for the $1^{\text {st }}, 2^{\text {nd }}$ and $3^{\text {rd }}$ evaluations.

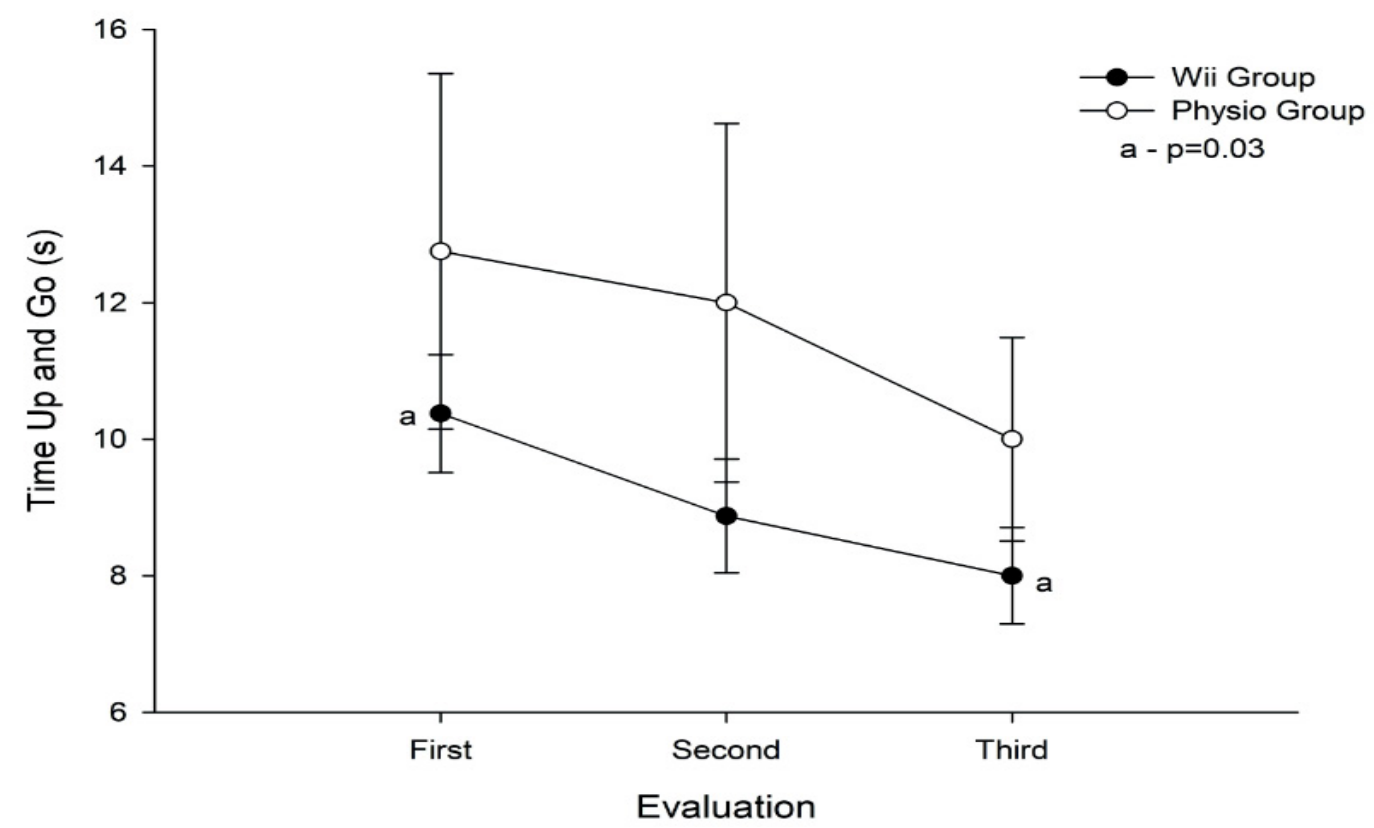

Figure 3: Time in seconds in the "time up and go" test, for the Wii-based rehabilitation and conventional physiotherapy groups, for the $1^{\text {st }}, 2^{\text {nd }}$ and $3^{\text {rd }}$ evaluations.

The Wii-based rehabilitation group exhibited significantly higher scores than the conventional physiotherapy group.

Our study shows that the Berg balance scale scores indicate an improvement in the patient balance after treating the patient with Wii-based rehabilitation. These data support the findings of a study by Coutinho and Matos [27] and Bateni [28], in which the participants who used the Nintendo ${ }^{\circledast}$ Wii Fit exhibited significant improvements in balance, as measured by using the Berg balance scale. Several studies indicate that training programs that use the Wii console lead to the improvement or maintenance of balance, by promoting corporal oscillations in various planes of movement, in a dynamic and articulated manner with visual and auditory feedback, which probably stimulates several proprioceptors [29]. Training with visual feedback 


\begin{tabular}{|c|c|c|c|c|c|c|c|c|}
\hline \multirow[b]{2}{*}{ Evaluations } & \multicolumn{2}{|c|}{ SDap } & \multicolumn{2}{|c|}{ SDml } & \multicolumn{2}{|c|}{ RMSap } & \multicolumn{2}{|c|}{ RMSmI } \\
\hline & Wii Group & Fisio Group & Wii Group & Wii Group & Wii Group & Fisio Group & Wii Group & Fisio Group \\
\hline Frist & $0.21 \pm 0.6$ & $0.71 \pm 1.1$ & $0.27 \pm 0.15$ & $0.15 \pm 0.9$ & $3.30 \pm 1.72$ & $3.15 \pm 2.58$ & $2.59 \pm 1.17$ & $2.71 \pm 0.81$ \\
\hline Second & $0.60 \pm 0.5$ & $0.74 \pm 1.0$ & $0.13 \pm 0.81$ & $0.11 \pm 0.9$ & $3.65 \pm 1.72$ & $3.58 \pm 1.68$ & $2.60 \pm 0.99$ & $2.79 \pm 1.53$ \\
\hline \multirow[t]{2}{*}{ Third } & $0.68 \pm 0.6$ & $0.9 \pm 1.02$ & $0.13 \pm 0.69$ & $0.13 \pm 0.2$ & $3.69 \pm 1.4$ & $3.73 \pm 1.03$ & $2.56 \pm 0.84$ & $2.49 \pm 0.89$ \\
\hline & \multicolumn{2}{|c|}{ AdCPap } & \multicolumn{2}{|c|}{ AdCPml } & \multicolumn{2}{|c|}{ TAS } & & \\
\hline Evaluations & Wii Group & Fisio Group & Wii Group & Wii Group & Wii Group & Fisio Group & & \\
\hline Frist & $7.55 \pm 3.68$ & $3.69 \pm 4.71$ & $2.10 \pm 0.37$ & $1.78 \pm 0.39$ & $1.59 \pm 0.52$ & $1.73 \pm 0.80$ & & \\
\hline Second & $3.40 \pm 3.00$ & $3.82 \pm 4.72$ & $1.79 \pm 0.97$ & $1.63 \pm 0.59$ & $1.63 \pm 0.30$ & $1.51 \pm 0.47$ & & \\
\hline Third & $3.89 \pm 5.59$ & $4.65 \pm 5.00$ & $1.38 \pm 0.55$ & $1.74 \pm 0.21$ & $1.73 \pm 0.61$ & $1.60 \pm 0.67$ & & \\
\hline
\end{tabular}

Table 1: Results of the the stabilometric data: Dispersion of the Center of Pressure displacement from the mean position in the anteroposterior direction (SDap); Dispersion of the Center of Pressure displacement from the mean position in the mediolateral direction (SDml); Root mean square displacement in the anteroposterior direction (RMSap); Root mean square displacement in the mediolateral direction (RMSml); Maximal and minimal CP displacements in the anteroposterior direction (AdCPap). Maximal and minimal CP displacements in the mediolateral direction (AdCPml); Total average speed (TAS). Results were not considered statistically significant. The results stabilometric data were provided for: SDap and SDml in cm/s; RMSap and RMSml in cm; AdCPap and AdCPml in cm; TAS in cm/s.

promotes motor learning and improves motor control capability through constant self-correction. During training with visual feedback by using the Nintendo ${ }^{\circledR}$ Wii, a series of motor tasks were proposed, in which motor planning skills and motor control were constantly stimulated [30]. Furthermore, Mao et al. [31] suggests that balance training with the Nintendo Wii can provide a more realistic visual and proprioceptive input, improving the patient's reaction time, postural stability and balance.

Additionally, we found with the functional reach test that only the group receiving the Nintendo ${ }^{\circledR}$ Wii treatment exhibited a statistically significant difference $(\mathrm{p}=0.01)$ between the first and third evaluations. Functional reach was higher in the third evaluation by $6 \mathrm{~cm}$. This finding was similar to the conclusions of Soares et al. [32], where the participants who underwent biofeedback training exhibited significant and lasting improvements in balance, as measured by using the same test. According to Tang and Fu [33] training with Wii Fit is effective because resembles natural body movements within the limits of stability.

Analyzing the TUG test results, we also found that only for the Wii-based rehabilitation group there was with a statistically significant improvement $(\mathrm{p}<0.01)$ by the end of the third evaluation. These participants exhibited a reduction of $2 \mathrm{sec}$ in their task performance time. This difference of $2 \mathrm{~s}$ represented a decrease in average speed of $0.18 \mathrm{~m} / \mathrm{s}$ from the first and last evaluation, which according to Nicholson et al. [34] has functional significance. Shorter time in the TUG test indicates that the Wii-based rehabilitation treatment promote improvement in dynamic balance. According to Prado et al. [35] the shorter TUG times require, primarily, muscle strength and balance, thus suggesting that the elderly who received the Wii treatment experienced an improvement in their muscle strength. Similarly, Mussato et al. [36] and Coutinho et al. [27] found shorter TUG times for participants who underwent treatment with Nintendo ${ }^{\circledR}$ Wii. Balance improvement with the Wii-based rehabilitation treatment was related to a higher and better use of the somatosensory and visual systems, as well as linear and angular acceleration information of the vestibular system [37].

For all of the stabilometric parameters analyzed no statistically significant difference was observed, both between the conventional physiotherapy and Wii-based rehabilitation groups, as well as between evaluations. These findings are in line with the study by Mussato et al. [36], who did not observe differences in anteroposterior, mediolateral and oscillation surface areas after the intervention with Wii Fit, in healthy elderly subjects. Also, according to these authors, who evaluated only AdCPap and AdCPml, the lack of statistically significant difference was due to the fact that $\mathrm{CP}$ oscillations were considered relative to their peak values, and not relative to the average oscillation amplitude of each individual, which might not be sufficiently sensitive for detecting differences among individuals. Freitas [38] offered an alternative explanation, suggesting that the lack of statistically significant difference could be attributed to the short time span of the evaluation.

\section{Conclusions}

We conclude that the Wii-based rehabilitation treatment was more effective in improving balance functional parameters than a more traditional physiotherapy protocol for older adults. Furthermore, the present study highlights the easy and simple applicability of the Nintendo ${ }^{\circledR}$ Wii as a resource for physiotherapeutic treatment. The Wii-based treatment is beneficial to the physiotherapeutic practice because in addition of being effective in improving patients' physical condition, it also offers a high degree of motivation during therapy, with consequent increased patient involvement. In addition, this treatment provides an opportunity for the continued treatment at home, since the Nintendo ${ }^{\circledR}$ Wii system is user-friendly and cost-efficient. We conclude that the Nintendo WII video game can be used as a powerful tool for physiotherapy balance disorders training.

\section{Acknowledgments}

Supported by Fapesp Processo 2012/09400-9 to Osmar Pinto Neto.

\section{References}

1. Matsumura BA, Ambrose AF (2006) Balance in the elderly. Clin Geriatr Med 22: $395-412$

2. Van Ooteghem K, Frank JS, Allard F, Buchanan JJ, Oates AR, et al. (2008) Compensatory postural adaptations during continuous, variable amplitude perturbations reveal generalized rather than sequence-specific learning. Exp Brain Res 187: 603-611.

3. Tainaka K, Takizawa T, Katamoto S, Aoki J (2009) Six-year prospective study of physical fitness and incidence of disability among community-dwelling Japanese elderly women. Geriatr Gerontol Int 9: 21-28.

4. FIDAN, Vural. (2012) The Role of Electronystagmography in Determining the Etiology of Vertigo. 2: 20-26

5. Melzer I, Kurz I, Oddsson LI (2010) A retrospective analysis of balance control parameters in elderly fallers and non-fallers. Clin Biomech 25: 984-988.

6. Goble DJ, Coxon JP, Wenderoth N, Van Impe A, Swinnen SP (2009) Proprioceptive sensibility in the elderly: degeneration, functional consequences and plastic-adaptive processes. Neurosci Biobehav Rev 33: 271-278.

7. Mancini M, Horak FB (2010) The relevance of clinical balance assessment tools to differentiate balance deficits. Eur J Phys Rehabil Med 46: 239-248.

8. de Noronha RDF, de Souza Vale RG, Giani TS, Bacellar S, Escobar T, et al. (2011) Correlation between static balance and functional autonomy in elderly women. Arch Gerontol Geriatr 52: 111-114. 
Citation: do Couto JPA, do Nascimento ML, Neto OP (2016) Comparative Study Using Functional and Stabilometric Evaluation of Balance in Elderly Submitted to Conventional Physiotherapy and Wii-Rehabilitation. Physiother Rehabil 1: 109. doi: 10.4172/2573-0312.1000109

Page 6 of 6

9. Souza DOR, Silva VF (2006) A importância do treinamento metal na reorganização do equilíbrio corporal em gerontes. Fitness and Performance J 5: 91-94.

10. Vaghetti CAO, Botelho SS, DAC (2009) Virtual learning environments in physical education: a review of the use of exergames. Science and Cognition 15: 64-75.

11. Butler DP, Willett $\mathrm{K}(2010)$ Wii-habilitation: is there a role in trauma? Injury 41 883-885

12. Deutsch JE, Borbely M, Filler J, Huhn K, Guarrera-Bowlby P (2008) Use of a low-cost, commercially available gaming console (Wii) for rehabilitation of an adolescent with cerebral palsy. Phys Ther 88: 1196-1207.

13. Shih $\mathrm{CH}$, Chang ML, Shih CT (2010) A new limb movement detector enabling people with multiple disabilities to control environmental stimulation through limb swing with a gyration air mouse. Res Dev Disabil 31: 875-880.

14. Bechara FT, Santos SMS (2008) Effectiveness of A physical therapy program for Balancing Training in Elderly. Magazine Health Research 1:15-20.

15. Wolf B, Feys H, Weerdt W (2001) Effect Of A Physical Therapeutic Intervention for Balance Problems in the Elderly: A Single-Blind, Randomized, Controlled Multicentre Trial. Clin Rehab 15: 624-636.

16. Barnett A, Smith B, Lord SR, Williams M, Baumand A (2003) Community-based group exercise improves balance and reduces falls in at-risk older people: randomised controlled trial. Age Ageing 32: 407-414.

17. Robertson MC, Devlin N, Gardner MM, Campbell AJ (2001) Effectiveness and Economic Evaluation of a Nurse Delivered Home Exercise Programme to Prevent Falls: 1 Randomised Controlled Trial. J Epidemiol Community Health 322: 697-702.

18. Soares MA, Sacchelli T (2008) Efeitos da Cinesioterapia no Equilíbrio de Idosos Rev Neurocienc 16: 97-100.

19. Silsupadol P, Siu KC, Shumway-Cook A, Woollacott MH (2006) Training of balance under single- and dual-task conditions in older adults with balance impairment. Phys Ther 86: 269-281.

20. Hu MH1, Woollacott MH (1994) Multisensory training of standing balance in older adults: I. Postural stability and one-leg stance balance. J Gerontol 49: M52-61.

21. Dean CM, Rissel C, Sharkey M, Sherrington C, Cumming RG, et al. (2009) Exercise Intervention To Prevent Falls and Enhance Mobility In Community Dwellers After Stroke: A Protocol For A Randomised Controlled Trial. BMC Neurol 9: 38-43.

22. Gardner MM, Robertson MC, Campbell AJ (2000) Exercise in preventing falls and fall related injuries in older people: a review of randomised controlled trials. Br J Sports Med 34: 7-17

23. Martins MSE (2010) Efficiency stabilometry and static baropodometry in balance assessment in patients with vestibular disorders. Dissertation (Masters in Health Sciences) - University of Brasilia, Faculty of Health Sciences 1-69.

24. Bogle Thorbahn LD, Newton RA (1996) Use of the Berg Balance Test to predict falls in elderly persons. Phys Ther 76: 576-583.

25. Duncan PW, Weiner DK, Chandler J, Studenski S (1990) Functional reach: a new clinical measure of balance. J Gerontol 45: M192-197.

26. Bohannon RW (2006) Reference values for the timed up and go test: a descriptive meta-analysis. J Geriatr Phys Ther 29: 64-68.

27. Coutinho AJAP, Matos TPG (2014) A Protocol Effects Assessment with Nintendo Platform $\mathrm{Wii}^{\circledR}$ in Cognitive and Physical Dimensions (Balance and Coordination) In Elderly. Psychology Journal 1: 55-62.

28. Bateni $H$ (2012) Changes in balance in older adults based on use of physical therapy vs the Wii Fit gaming system: a preliminary study. Physiotherapy 98 211-216

29. Rojas VG, Cancino EE, Silva CV, López MC, Arcos JF (2010) Impact balance training through virtual reality in a population of older adults. Intl J Morphol 28 : 303-308.

30. Cho SH, Shin HK, Kwon YH, Lee MY, Lee YH, et al. (2007) Cortical activation changes induced by visual biofeedback tracking training in chronic stroke patients. NeuroRehabilitation 22: 77-84

31. Mao Y, Chen P, Li L1, Huang D1 (2014) Virtual reality training improves balance function. Neural Regen Res 9: 1628-1634.

32. Soares AV, Hochmuller ACOL, Silva P, Fronza D, Woellner SS, et al. (2009) Biofeedback for balance training in hemiparesis from stroke: a preliminary study. Fisioter Pesqui 16:132-136.

33. Tsang WW, Fu AS (2016) Virtual reality exercise to improve balance control in older adults at risk of falling. Hong Kong Med J 22 Suppl 2: 19-22.

34. Nicholson VP, McKean M, Lowe J, Fawcett C, Burkett B (2015) Six weeks of unsupervised Nintendo Wii Fit gaming is effective at improving balance in independent older adults. J Aging Phys Act 23: 153-158.

35. Prado RA, Teixeira ALC, Langa CJSO, Egydio PRM, Izzo P (2010) The influence of resistance exercise in balance, functional mobility and quality of life of elderly . World Health 34: 183-191.

36. Mussato R, Brandalize D, Brandalize M (2012) Nintendo Wii ${ }^{\circledR}$ and its effect on balance and functional capacity of healthy elderly. $\mathrm{R}$ Bras $\mathrm{Ci}$ and Mov 20: 68-75.

37. Araujo TB, Silva NA, Costa JN, Pereira MM, Safons MP (2011) Effect of equineassisted therapy on the postural balance of the elderly. Rev Bras Fisioter 15 414-419.

38. Freitas JP (2003) Behavioral characteristics of postural control of young people, adults and seniors. Dissertation (Masters in Physical Education) - Institute of Biosciences, University Paulista- (Unesp), Rio Claro. 